Rios, R. (2018). Formación para la innovación en los programas de Contaduría Pública. Estudio de caso. Contaduría Universidad de Antioquia, 73, 79-101.

Doi: https://doi.org/10.17533/udea.rc.n73a04

\title{
Formación para la innovación en los programas de Contaduría Pública. Estudio de caso*
}

Roberto Antonio Ríos León

riosleonroberto@gmail.com

Uniminuto

Una primera versión de este trabajo fue presentada en el III Congreso Latinoamericano de Investigadores Contables, Medellín. Del 19 al 21 de abril de 2018. 
Formación para la innovación en los programas de Contaduría Pública. Estudio de caso.

Resumen: La presente investigación se desarrolló en Bogotá, D.C., Colombia, en el año 2017, con el objetivo de determinar la incidencia de la formación para la innovación en el desempeño académico de los estudiantes de Contaduría Pública. Para ello se inició con la revisión bibliográfica para establecer los referentes teóricos y el estado del arte relacionados con formación e innovación. A continuación, se aplicaron 221 encuestas, se realizó un grupo de discusión y 6 entrevistas semiestructuradas, para luego analizar los datos mediante la utilización del software estadístico SPSS, el proceso de destilación de información y la triangulación de datos, para finalmente determinar las conclusiones. Como resultado se evidenció la necesidad de fortalecer los programas académicos de Contaduría Pública, en lo relacionado con la formación para la innovación, para que los estudiantes potencien su desempeño académico mediante la adquisición de conocimientos, habilidades y destrezas en la disciplina de la innovación.

Palabras clave: Formación, innovación, formación para la innovación, desempeño académico.

Training for innovation in the Public Accounting programs. Case study.

Abstract: The present research was developed in the city of Bogotá, Colombia, in the year 2017, with the aim to determine the incidence of training for innovation on the academic performance of Public Accounting students. To do so, a bibliographic review was first conducted in order to establish the theoretical references and the state of the art related to training and innovation. Subsequently, 221 surveys were applied, a discussion group and 6 semi structured interviews were carried out, to then analyze the data using the SPSS statistical software, the information distillation process and data triangulation, and finally determine the conclusions. As result, the need to strengthen the Public Accounting academic programs was evidenced, in relation to training for innovation, so that the students potentiate their academic performance through the acquisition of knowledge, abilities and skills in the innovation discipline.

Keywords: Training, innovation, training for innovation, academic performance.

Formation à l'innovation dans les programmes académiques de Comptabilité Publique. Étude de cas. Résumé: cette recherche a été menée à Bogotá, Colombie, en 2017, afin de déterminer l'incidence de la formation à l'innovation sur la performance académique des étudiants de la Comptabilité Publique. Pour ce faire, nous avons commencé par une analyse de la littérature afin d'établir les référents théoriques ainsi que l'état d'art liés à la formation et à l'innovation. Ensuite, nous avons fait un sondage avec 221 personnes, crée un groupe de discussion et menée six entretiens semistructurés, pour analyser les données grâce au logiciel d'analyse statistique SPSS, effectuer la distillation de l'information et la triangulation de données, pour arriver finalement aux conclusions. Tout ceci a mis en évidence le besoin de consolider les programmes académiques de Comptabilité Publique en ce qui concerne la formation à l'innovation, afin que les étudiants renforcent leur performance académique au moyen de l'acquisition des connaissances, compétences et du savoirfaire de la discipline de l'innovation.

Mots-clés: formation, innovation, formation à l'innovation, performance académique.

Formação para a inovação nos programas de Contabilidade Pública. Estudo de caso.

Resumo: Esta pesquisa desenvolveu-se em Bogotá, D.C., Colômbia, no ano 2017, com o intuito de determinar a incidência da formação para a inovação no desempenho acadêmico dos estudantes de Contabilidade Pública. Para tal fim, iniciou-se com a revisão bibliográfica para estabelecer os referentes teóricos e o estado da arte relacionados com formação e inovação. A seguir, fizeramse 221 enquetes, realizou-se um grupo de discussão e 6 entrevistas semiestruturadas, para depois analisar os dados através da utilização do software estatístico SPSS, o processo de "destilação de informação" e a triangulação de dados, para finalmente determinar as conclusões. Como resultado, evidenciou-se a necessidade de fortalecer os programas de Contabilidade Pública no relacionado com a formação para a inovação, para que os estudantes possam potenciar seu desempenho académico através da aquisição de conhecimentos, habilidades e destrezas na disciplina da inovação.

Palavras chave: Formação, inovação, formação para a inovação, desempenho académico. 


\title{
Formación para la innovación en los programas de Contaduría Pública. Estudio de caso.
}

\author{
Roberto Antonio Ríos León \\ Doi: https://doi.org/10.17533/udea.rc.n73a04
}

Primera versión recibida en julio de 2018 - Versión final aceptada en agosto de 2018

\section{Introducción}

E

n Buenos Aires, Argentina, entre el 2 y el 4 de octubre de 1995, se llevó a cabo la Conferencia Científica de la V Cumbre Iberoamericana de Jefes de Estado y de Gobierno de la Organización de Estados Iberoamericanos, en la cual el tema fue la "Formación para la Innovación". Como resultado de esta conferencia, se generaron conclusiones y recomendaciones relacionadas con la investigación, el desarrollo y la innovación.

De particular relevancia para la formación innovadora es su conclusión de que la enseñanza - aprendizaje requiere de métodos, técnicas e instrumentos para aprender a pensar, para aprender a aprender y a hacer. Ahora bien, si se retoma el papel de las instituciones que imparten la formación para la innovación, haciendo énfasis en la Universidad, es claro que aún falta mucho por hacer, y en consecuencia una de las grandes apuestas es la formación del talento humano en temas de innovación.

Por otro lado, la Organización de las Naciones Unidas (ONU), en la Cumbre para el Desarrollo Sostenible llevada a cabo en septiembre de 2015, estableció la Agenda 2030 para el Desarrollo Sostenible (conocida también como Agenda de Desarrollo Post- 2015), y en la cual se definieron 17 Objetivos de Desarrollo Sostenible, que contemplan la innovación como motor de crecimiento y desarrollo económico.

En este sentido, y de acuerdo con el objetivo número 9: Industria Innovación e Infraestructura, la ONU considera que se deben construir infraestructuras resilientes, promover la industrialización inclusiva y sostenible y fomentar la innovación, como motores fundamentales del crecimiento y del desarrollo económico. 
La problemática anteriormente expuesta se plantea a través de la siguiente pregunta de investigación: ¿Los estudiantes de Contaduría Pública mejoran su desempeño académico potenciando su formación para la innovación?

Para dar respuesta a la pregunta de investigación planteada se definió como objetivo general, establecer cómo se lleva a cabo la formación para la innovación en los programas de Contaduría Pública analizando la eficacia de las acciones formativas, para determinar su incidencia en el desempeño académico de los estudiantes.

Para dar cumplimiento al objetivo general, se definieron como objetivos específicos:

- Identificar las características de la formación para la innovación en los programas de Contaduría Pública.

- Analizar la eficacia de las acciones formativas en innovación.

- Determinar la incidencia de la formación para la innovación en el desempeño académico de los estudiantes del programa de Contaduría Pública.

\section{Referentes teóricos}

\section{II.1 Formación}

En el diccionario de la Real Academia Española (2016) se define formar como "preparar intelectual, moral o profesionalmente a una persona o grupo de personas", en tanto que formación la define como "acción y efecto de formar o formarse". Entonces se considera como formación tanto el adiestramiento como el resultado de este, y se adquiere a través del proceso de aprendizaje.

Formación, de acuerdo con Álvarez y Rubio $(2010$, p.20) se define como "un término inequívoco por el que se entiende tanto el adiestramiento como el resultado de este; y debe diferenciarse de conceptos concomitantes, como aprendizaje, enseñanza y educación". Por lo tanto, la formación puede definirse como el esfuerzo sistemático y planificado dirigido a la modificación o desarrollo de conocimientos, técnicas y actitudes, a través de la experiencia de aprendizaje y a conseguir la actuación adecuada en una actividad o rango de actividades.

La formación es entendida igualmente como el conjunto de enseñanzas impartidas diseñadas para dirigir al alumno hacia las tareas operativas del ámbito laboral, es decir las que requieren una capacitación más práctica, específica y fácilmente demostrable (Álvarez y Rubio, 2010), en contraposición a la educación que lejos de capacitar en habilidades concretas estima la puesta a prueba de una disciplina y la capacidad de comprensión global de la sociedad, por medio de la adquisición de conocimientos teóricos. 
En términos generales, la formación es entendida como el conjunto de enseñanzas impartidas que permiten obtener conocimientos, desarrollar actitudes y adquirir destrezas para asumir una tarea o para cumplir un objetivo. En este sentido, se trata de un proceso que requiere un objeto de estudio, unas estrategias de enseñanza aprendizaje, un sujeto dispuesto a aprender, un maestro dispuesto a enseñar y un entorno adecuado, para el caso, las instituciones de educación superior.

En el proceso de formación, de acuerdo con José Tejada Fernández (2001, p 111), catedrático de didáctica de la Universidad Autónoma de Barcelona "El profesor tiene un papel determinante en el proceso de enseñanza-aprendizaje (en este caso en temas de innovación) ya que es un mediador entre el currículum y sus destinatarios, entendiendo que estos destinatarios son también mediadores de su propio aprendizaje".

En síntesis, (Tejada Fernández, 2001, p 124), "el profesor de hoy, por las exigencias de su práctica, es un profesional que toma decisiones, flexible, libre de prejuicios (actitud de anteponerse y rectificar a tiempo), comprometido con su práctica" (reflexiona sobre la misma y aporta elementos de mejora); en consecuencia, el perfil del nuevo profesor debe caracterizarse por:

- Espíritu innovador;

- Flexibilidad;

- Trabajo en equipo;

- Conocimientos tecnológicos;

- Cree en su profesión;

- Sentido de responsabilidad y compromiso.

Desde esta perspectiva, le es propio al profesor de hoy y seguramente al profesor del mañana:

Innovar para formar: que significa disponer de los conocimientos, habilidades y actitudes de mejora profesional permanente para optimizar el proceso de enseñanza aprendizaje.

Formar para innovar: que equivale a aprender, es decir capacitar para introducir cambios y mejorar procesos de enseñanza aprendizaje.

\section{II.2 Innovación}

En el diccionario de la Real Academia Española se define Innovar como "Mudar o alterar algo, introduciendo novedades", en tanto que innovación la define como "acción y efecto de innovar, creación o modificación de un producto y su introducción en un mercado".

Drucker, P. (1986, p. 31) define la innovación como "La herramienta específica de los empresarios innovadores; el medio con el cual explotar el cambio como una oportunidad para un negocio diferente. Se la presenta como una disciplina que puede aprenderse y practicarse". 
Rios, R. Formación para la innovación en los programas de Contaduría Pública. Estudio de caso

Según el Manual de Oslo (2006)

Una innovación es la introducción de un nuevo, o significativamente mejorado, producto (bien o servicio), de un proceso, de un nuevo método de comercialización o de un nuevo método organizativo, en las prácticas internas de la empresa, la organización del lugar de trabajo o las relaciones exteriores. (p. 56)

Para Hamel, G., (2008, p.23) "La innovación administrativa es cualquier cosa que modifique sustancialmente la manera como se administra, o que modifique ostensiblemente las formas habituales de organización y, con ello, promueva los fines de la empresa".

Ponti, F., (2010, p. 40) define que "Innovar significa alterar los procesos inteligentes de una empresa (...) para que sea capaz, de forma regular de generar output de innovación (productos, servicios, estrategias), que aumenten la rentabilidad y generen una mayor fidelidad de los clientes implicados".

Nieto, J., (2012, p. 53) considera que "La innovación se materializa en un proceso, o camino que, haciendo algo original o que no se hubiese hecho anteriormente, intenta conseguir un fin mejor a través del uso del conocimiento".

Así como hay múltiples definiciones de innovación, algunas expresadas en lenguaje específico y otras en lenguaje genérico, quizá las más comprensible para quienes se inician en este mundo de la innovación es la presentada por Gallo, C., (2011, p. xv) "La innovación es una nueva manera de hacer cosas, que resulta en un cambio positivo", que hace mejor la vida.

Se entiende por innovación en sentido general como el aprovechamiento de una oportunidad que genera una novedad que, al ser explotada, genera ventajas competitivas o beneficios para quien la realiza. En este orden de ideas, existen diversas definiciones de innovación, clasificaciones y formatos que atienden a las características propias de la innovación y la forma en que esta se da, al igual que a su propósito.

\section{II.3 La formación para la innovación}

De acuerdo con Pineda, P., Duran, M., Ciraso, A., Espona, B. (2015), es pertinente dar claridad conceptual a dos definiciones: formación innovadora, que hace referencia a una formación que por su metodología se considera innovadora porque en ella se utilizan quizá nuevas tecnologías de la información y recursos alejados de la formación tradicional y formación para la innovación que hace referencia a aquella formación que pretende generar innovaciones en las organizaciones.

Por lo anterior, no se puede concluir que un proceso de formación con aplicación de metodologías innovadoras genere innovación en las personas y en las organizaciones, se puede dar el caso, pero no es frecuente que así suceda. 


\section{II.3.1 La enseñanza y la cultura de la innovación.}

De acuerdo con la Organización de Estados Iberoamericanos (OEI, 1995), es fundamental educar para la capacitación en la generación de soluciones útiles y prácticas, tanto manuales como conceptuales y una visión holística e integradora de conceptos, en lugar de las antiguas enseñanzas atomizadas e impartidas desde una perspectiva autoritaria, formalista, acrítica y meramente retransmisora de conocimientos.

Lograr esto pasa necesariamente, por la cualificación de cientos de miles de profesores y por una fuerte inversión de recursos en la generación y difusión de investigaciones educativas de carácter teórico, experimental y aplicado (OEI, 1995). Para potenciar la innovación no sólo se requiere educar a los individuos, sino también proporcionarles el entorno institucional adecuado. En consecuencia, es un prerrequisito indispensable para el despliegue del potencial creativo, la generación de un contingente importante de profesionales que reúnan los conocimientos, habilidades y experiencias, como para liderar el proceso innovador.

La gestión de la innovación es, por decirlo así, una ciencia en el limbo en Iberoamérica (OEI, 1995), a pesar de que, en el mundo industrializado es una materia objeto de numerosas publicaciones periódicas y uno de los temas más candentes de la producción académica y bibliográfica. No existen en muchos países, textos, docentes ni material educativo de pregrado aplicable a la enseñanza de este tema.

\section{II.3.2 Recomendaciones para la formación en innovación}

Tomando como referencia las recomendaciones de la Conferencia Científica de la V Cumbre Iberoamericana de Jefes de Estado y de Gobierno - realizada en Buenos Aires (Argentina), del 2 al 4 de octubre de 1995-, cuyo tema fue la "Formación para la Innovación", a continuación, se exponen los principales aspectos relacionados con la formación para la innovación y que tienen que ver con los gobiernos, las instituciones educativas y el sector empresarial.

\section{Recomendaciones específicas a los gobiernos}

- Incorporar la cultura de la innovación en el aparato estatal, como agente de su promoción en la sociedad.

- Asumir un rol en el estímulo del clima de innovación en las empresas, incentivando la capacitación para la innovación.

- Garantizar un apoyo continuo a las instituciones educativas, científicas y tecnológicas.

- Promover y apoyar actividades de cooperación en los ámbitos de la formación para la innovación, la gestión tecnológica, la investigación científica, la experimentación e investigación educativa y la cooperación empresarial. 
- Asociar los apoyos gubernamentales a esquemas claros y explícitos de evaluación de indicadores sobre el desempeño institucional.

\section{Recomendaciones específicas a las instituciones educativas}

- Promover la cultura de la innovación en la sociedad, favoreciendo una formación apropiada en todos los niveles de enseñanza.

- Revisar los conceptos en relación con los contenidos y formas de impartir la educación.

- Desarrollar un amplio programa de investigaciones y pruebas experimentales orientadas a la introducción de estos conceptos educativos.

- Introducir, en el currículum de diversas carreras profesionales, los conceptos y métodos de la gestión de la innovación.

- Fortalecer los Centros y programas de popularización de la ciencia y la tecnología.

\section{Recomendaciones específicas al sector empresarial}

- Promover, a través de las organizaciones empresariales, la difusión de la cultura de la innovación y las nuevas técnicas de gestión en las empresas.

- Incrementar la participación en las actividades de investigación y desarrollo, compartiendo el esfuerzo y el financiamiento de estas actividades.

- Colaborar con las instituciones educativas y de investigación en la definición de sus programas y en la concreción de sus requerimientos.

- Asumir de forma creciente su responsabilidad en la internacionalización de la economía de sus empresas y en las actividades de cooperación.

Ahora bien, en la economía del conocimiento, el talento humano es la principal materia prima, por tanto, hay que crear talento y actuar con talento. Así pues, la formación debe llevarse a cabo mediante la fijación de retos, los cuales activan al ser humano, y esta activación se hace efectiva mediante acciones para realizar.

Cuando se trata de innovación, hay que dejar de lado el ser anecdótico y pensar que las experiencias del pasado ratifican el futuro, ya que las historias matan las ideas, al igual que permiten construir muros, cuando la innovación requiere crear puentes.

Respecto a la formación para la innovación en el contexto de la universidad con relación a la empresa, es evidente una desconexión entre el mundo académico y el sector productivo, lo cual, según Arraut (2014), se debe a que en la educación universitaria hay carencias en el fortalecimiento del emprendimiento, la innovación y la creatividad como elementos esenciales en la formación de profesionales.

\section{II.3.3 Competencias en la formación de innovadores}

Hoy en día, se habla mucho sobre la necesidad de las escuelas de impulsar competencias del siglo XXI. La Sociedad para las Competencias del siglo XXI, 
con sede en los Estados Unidos, es un consorcio de diecinueve estados y treinta y tres empresas asociadas. Propugna un enfoque amplio del plan de estudios y el aprendizaje que engloba las siguientes categorías (Robinson, 2016):

\section{Temas interdisciplinarios:}

- Conciencia planetaria

- Cultura financiera, económica, comercial y empresarial.

- Cultura cívica

- Cultura ambiental

Competencias para el aprendizaje:

- Creatividad e innovación

- Pensamiento crítico y resolución de problemas

- Comunicación y colaboración

Competencias para la vida y el trabajo:

- Flexibilidad y adaptabilidad

- Iniciativa y autonomía

- Competencias sociales y transculturales

- Productividad y responsabilidad

- Capacidad de liderazgo y de responsabilidad

Las escuelas necesitan cultivar la gran diversidad de talentos e intereses de los jóvenes, eliminar las fronteras entre los estudios académicos y los técnicoprofesionales y conceder la misma importancia a las dos áreas de estudio, y finalmente fomentar asociaciones prácticas con el mundo laboral para que los jóvenes puedan experimentar en persona distintos tipos de entornos laborales.

De acuerdo con Pineda, et. al. (2015) las competencias principales que fomenta la formación para la innovación son:

- El pensamiento reflexivo sobre la innovación.

- La actitud innovadora relacionada con el espíritu emprendedor.

- La generación de ideas mediante la creatividad.

- La planificación de la innovación a partir de la generación de una idea.

- La comunicación.

La actitud positiva hacia la innovación, el cambio y la formación continua.

Para el desarrollo de la capacidad creativa de las personas, es importante tener presente que se den las condiciones mínimas para que ello sea posible, ya que la diferencia entre el éxito y el fracaso es quien se da por vencido primero.

Para crear o fortalecer las competencias en innovación, y de acuerdo con Pineda, et al. (2015) las estrategias que permiten potenciar esta formación son:

- Trabajo en equipo.

- Interdisciplinariedad.

- Combinación de métodos pedagógicos.

- Juegos como materiales de apoyo para aplicar conocimientos adquiridos.

- Experimentación. 
- Aprender de los errores.

- Uso de las tecnologías de la información y la comunicación.

- Atender las necesidades e intereses de los participantes y la organización.

Ahora bien, en los procesos de formación para la innovación se hace referencia a las habilidades de los innovadores. En este sentido, Wagner (2013), considera que las nuevas habilidades que todos los estudiantes necesitan para todas las carreras, el aprendizaje continuo y la ciudadanía, en un mundo cada vez más plano, son:

- El pensamiento crítico y la resolución de problemas.

- La colaboración a través de redes y el liderazgo por influencia.

- La agilidad y la adaptabilidad.

- La iniciativa y el espíritu emprendedor.

- El acceso y el análisis de la información.

- La comunicación efectiva oral y escrita.

- La curiosidad y la imaginación.

Las habilidades mencionadas, según Wagner (2013), son necesarias mas no suficientes; en este contexto la curiosidad y la imaginación son sin duda, fuentes de innovación. Igualmente se requiere de ciertas cualidades para ser innovador, tales como la perseverancia, la voluntad de experimentar, la asunción de riesgos calculados, la tolerancia al fracaso, la capacidad de diseñar el pensamiento, además del pensamiento crítico.

\section{Metodología de la investigación}

Tomando como punto de partida la revisión bibliográfica realizada, se llevó a cabo una investigación caracterizada por la utilización de metodologías con enfoque cuantitativo y cualitativo, es decir una metodología ecléctica, en función del problema y los objetivos de la investigación (Hernández Pina, 1997).

Para el enfoque cuantitativo, se ha propuesto una metodología descriptiva dentro de los modelos no experimentales, basados en la encuesta y más concretamente en la encuesta transversal mediante la aplicación de cuestionario (Anguera, 1995).

Para el enfoque cualitativo, se ha recurrido al estudio de caso (Colás, 1999). En el marco del estudio de caso, se emplearon las siguientes técnicas cualitativas de recolección de datos: entrevista semiestructurada, grupo de discusión y análisis documental utilizando para ello los guiones de acuerdo con cada técnica aplicada.

\section{III.1 Descripción de la población y muestra}

Bisquerra (1989, p. 81), define la población como "el conjunto de todos los individuos en los que se desea estudiar el fenómeno" y la muestra como "el subconjunto de la población sobre el cual se realizan las observaciones". 
La población la conformaron 1.200 estudiantes del programa académico de Contaduría Pública, ofertado por una Institución de Educación Superior, ubicada en Bogotá, D.C., cursando estudios en el año 2016. La población y la muestra, se reflejan en la tabla 1 .

Tabla 1: muestra obtenida para la investigación.

\begin{tabular}{lccccc}
\hline & Población & $\begin{array}{c}\text { Muestra } \\
\text { Invitada }\end{array}$ & $\begin{array}{c}\text { Muestra } \\
\text { Aceptante }\end{array}$ & $\begin{array}{c}\text { \% de la muestra } \\
\text { invitada }\end{array}$ & $\begin{array}{c}\text { \% de la } \\
\text { población }\end{array}$ \\
\hline Estudiantes & 1.200 & 240 & 221 & $92 \%$ & $18,5 \%$ \\
Total & 1.200 & 240 & 221 & $92 \%$ & $18,5 \%$ \\
\hline
\end{tabular}

Fuente: elaboración propia a partir de la investigación.

En total, la muestra invitada la conformaron 240 estudiantes, y la muestra aceptante con los mismos, 221 estudiantes, lo que corresponde a un $92 \%$ de la muestra invitada; respecto a la población, representa un $18,5 \%$. La selección de la muestra se realizó mediante muestreo aleatorio simple.

La caracterización de la muestra es la siguiente:

- Edad promedio: 22 años.

- Sexo: $52 \%$ masculino, $48 \%$ femenino.

- Cursos: Contabilidad financiera, Finanzas, Auditoria, Costos, Presupuestos y Tributaria, correspondientes a los semestres de $1^{\circ}$ a $9^{\circ}$.

La entrevista semiestructurada se aplicó a 5 informantes (directivos y profesores del programa académico), diferentes a los del grupo de discusión, atendiendo los criterios de conocimiento del programa académico, conocimiento de temas de innovación, experiencia en formación de innovadores. El grupo de discusión se llevó a cabo con 6 informantes (profesores del programa académico), atendiendo los mismos criterios de la entrevista semiestructurada. El análisis documental se realizó tomando el documento maestro del programa, el plan de estudios, los planes de curso relacionados con innovación y documentos relacionados con formación de innovadores.

\section{III.2 Instrumentos de recolección de información}

Los instrumentos utilizados para la recolección de información se agrupan en función del problema y los objetivos de la investigación. De este modo se tiene lo siguiente:

Para el estudio de encuesta se ha utilizado el cuestionario. El cuestionario es el instrumento de recolección de información más utilizado en la investigación por encuesta (Buendía, 1997). Para Martínez Arias (1995), es uno de los métodos más comunes para obtener datos estadísticos acerca de una gran variedad de temas, con propósitos de investigación, siendo aplicado en las distintas disciplinas. 
El cuestionario fue sometido a juicio de expertos para su validación, para ello se invitaron 4 expertos, mediante carta en la cual se indican los objetivos de la investigación. En este sentido, Fox (1981), manifiesta que la validez de contenido es la técnica más apropiada cuando se trata de validar cuestionarios. Respecto a la fiabilidad de los cuestionarios se ha establecido mediante procedimientos estadísticos utilizando el Alfa de Cronbach, obteniendo el siguiente resultado: 0.896. Del dato anterior se desprende que los resultados de la prueba aplicada, Alfa de Cronbach, al cuestionario son muy buenos, por lo que se concluye que el instrumento para la recogida de información es fiable.

Para el estudio de caso se ha contado con varias técnicas cualitativas: entrevista semiestructurada, grupo de discusión y análisis de documentos. La entrevista aporta la profundidad, el detalle y las perspectivas de los entrevistados (Del Rincón y otros, 1995). Respecto al grupo de discusión y según Colás Bravo (1999), corresponde a una técnica de investigación social que trabaja con el habla, descubriendo, a través de sus formas de lenguaje dimensiones socioculturales y cognitivas. Por su parte el análisis de documentos, permite profundizar en la comprensión del tema de investigación.

\section{III.3 Triangulación de los datos}

Colás (1999, p. 274) establece la triangulación "como el procedimiento mediante el cual se obtiene credibilidad en los resultados, es decir, se establece la consistencia entre los datos recogidos por el investigador y la realidad". Cohen y Manion (1990, p. 331), la definen como "el uso de dos o más métodos de recogida de datos en el estudio de algún aspecto". Para el caso presente se recurrió a la triangulación de núcleos combinados ya que se utilizó más de un nivel de análisis: nivel individual, nivel grupal y nivel de colectividades (Cohen y Manión, 1990).

\section{III.4 Tratamiento y análisis de los datos}

El análisis estadístico de los datos se realizó con el programa estadístico SPSS (Statistical Packege of Social Sciences) versión 19.0 para Windows. El proceso ha consistido en importar todos los datos, etiquetarlos y posteriormente realizar un análisis descriptivo. Para el tratamiento de los datos cualitativos, se analizaron utilizando para ello el registro en el procesador de textos Word (Paquete Microsoft Office XP), tomando como referencia el proceso de Destilar la Información (Vásquez, 2005).

\section{Resultados}

Los estadísticos utilizados en el análisis de las variables del cuestionario corresponden a porcentajes. A continuación, se presenta el resumen de los estadísticos descriptivos del cuestionario, correspondientes a la formación para la innovación en el programa académico, expresados en figuras y 
porcentajes para cada una de las variables. Los estadísticos se complementan con los resultados del análisis cualitativo, expresados mediante citas textuales obtenidas de las entrevistas semiestructuradas y los grupos focales, que dan mayor sentido al análisis realizado.

IV.1 Grado de conocimiento del modelo de innovación del programa

El $48,87 \%$ considera que el grado de conocimiento del modelo es bajo; el $27,60 \%$, que es medio; el $23,08 \%$, que es nulo y el $0,45 \%$ que es alto (figura 1 ).

Figura 1. Grado de conocimiento respecto al modelo de innovación del programa.

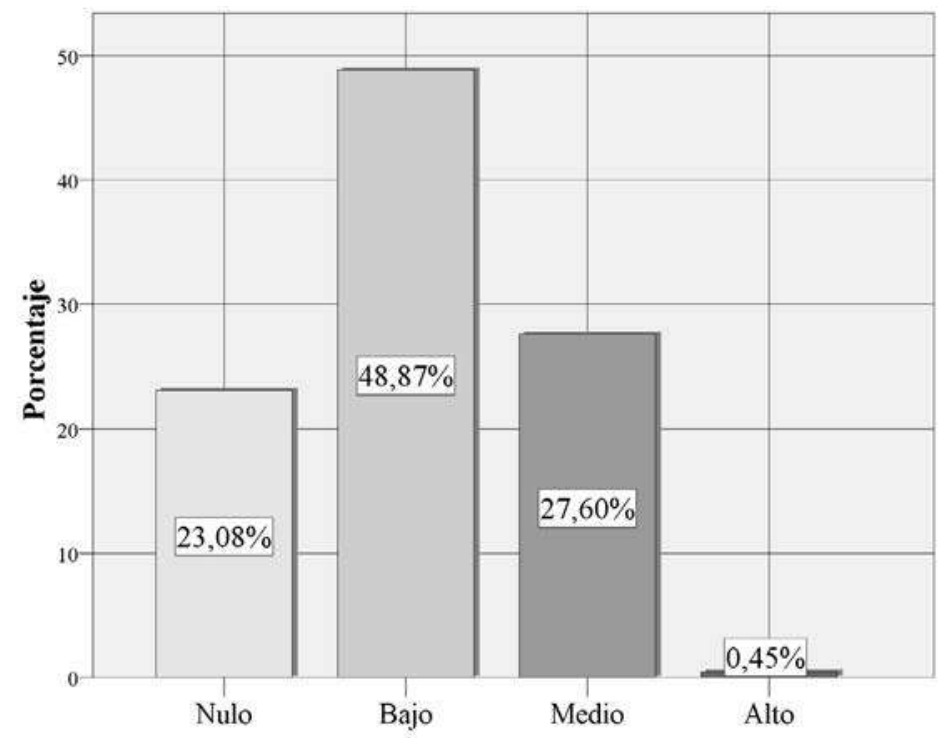

Fuente: elaboración propia a partir de los resultados de la investigación.

El programa académico tiene como uno de sus propósitos formar profesionales innovadores y transformadores de la realidad social de su contexto; sin embargo, no especifica la forma como se está llevando a cabo esta formación en innovación, lo que explica el bajo grado de conocimiento respecto al modelo de innovación del programa. Tradicionalmente se ha considerado que la innovación es propia de la industria y no ha tenido un desarrollo pleno en la universidad, aunque se evidencian algunos esfuerzos para abordar este asunto en los procesos de formación.

R1. GD PA1. "El modelo de innovación asociado a la industria se basa en un esquema cerrado en el que el proceso de innovar está monopolizado por la empresa, y no trasciende fácilmente el mundo universitario". R1. ES PP1. “(...) la innovación la veo en la forma como la cátedra debe participar en la comunidad (...)". 


\section{IV.2 Grado de conocimiento respecto a la formación para la innovación del programa}

En cuanto al grado de conocimiento respecto a la formación para la innovación del programa, el 51,58\% manifiesta que es bajo; el $23,98 \%$, que es nulo; el 23,98\%, que es medio, y el 0,45, que es alto (figura 2).

Figura 2. Grado de conocimiento respecto a la formación para la innovación del programa.

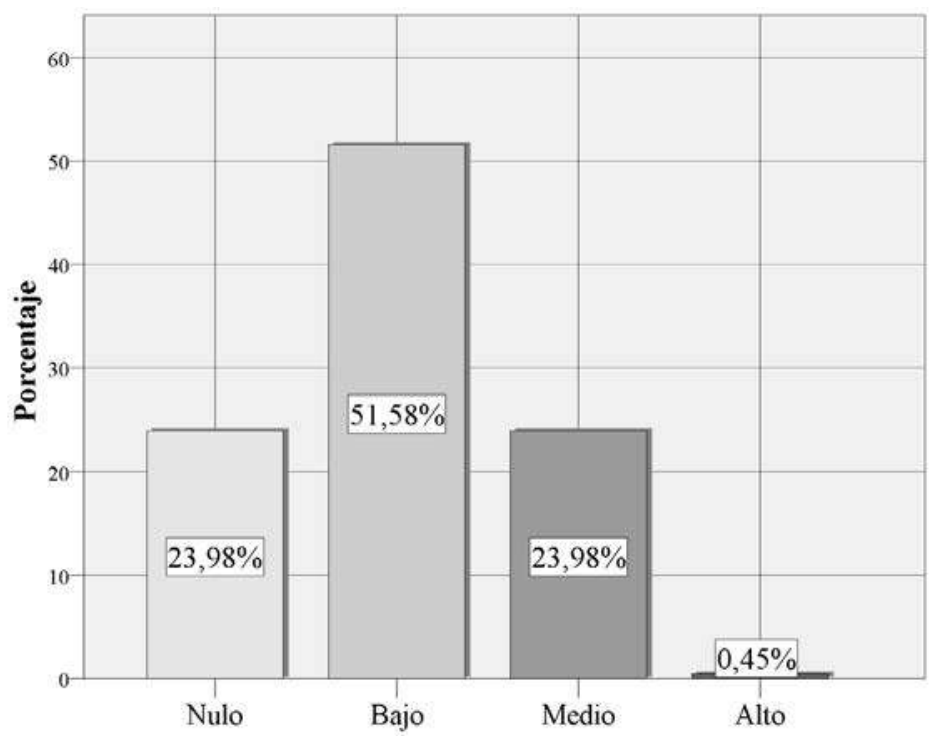

Fuente: elaboración propia a partir de los resultados de la investigación.

El grado de conocimiento es bajo, ya que en el programa se habla poco de innovación y mucho de investigación. A pesar de ello, hay un gran interés por el tema, lo cual indica que se debe socializar con mayor frecuencia y profundidad en materia de innovación en el programa. Esto implica hacer explícitos lo propósitos de formación mediante la actualización de los planes de estudio y los planes de curso.

R2. ES DP1. "He tenido más que todo experiencia en temas de investigación, pero el tema de innovación lo he escuchado, me parece un tema supremamente interesante (...)". R9. ES DP1. "Por supuesto, ese es un tema futurista que debemos de abordar y que no debemos de dejar pasar (...) tenemos que incluirlo entre nuestros currículos,". R1. ES PE2. "no es usual encontrar referentes teóricos relacionados con la formación para la innovación”. R1. GD PN1. "Quizá innovamos a diario, pero no somos conscientes de ello, y tomar consciencia es tener conocimiento, y en este caso, de innovación, y se piensa 
en tecnología y no en conocimiento”. R19. ES DP1. “(...) mediante una electiva, porque podemos involucrar estudiantes de diferentes áreas de formación”.

IV.3 Acerca de si el programa cuenta con un plan que oriente la formación para la innovación

Según el 66,52\%, algunas veces; el 22,62\% considera que nunca; el 9,50\%, que muchas veces, y el 1,36\%, que siempre (figura 3 ).

Figura 3. El programa cuenta con un plan que oriente la formación para la innovación.

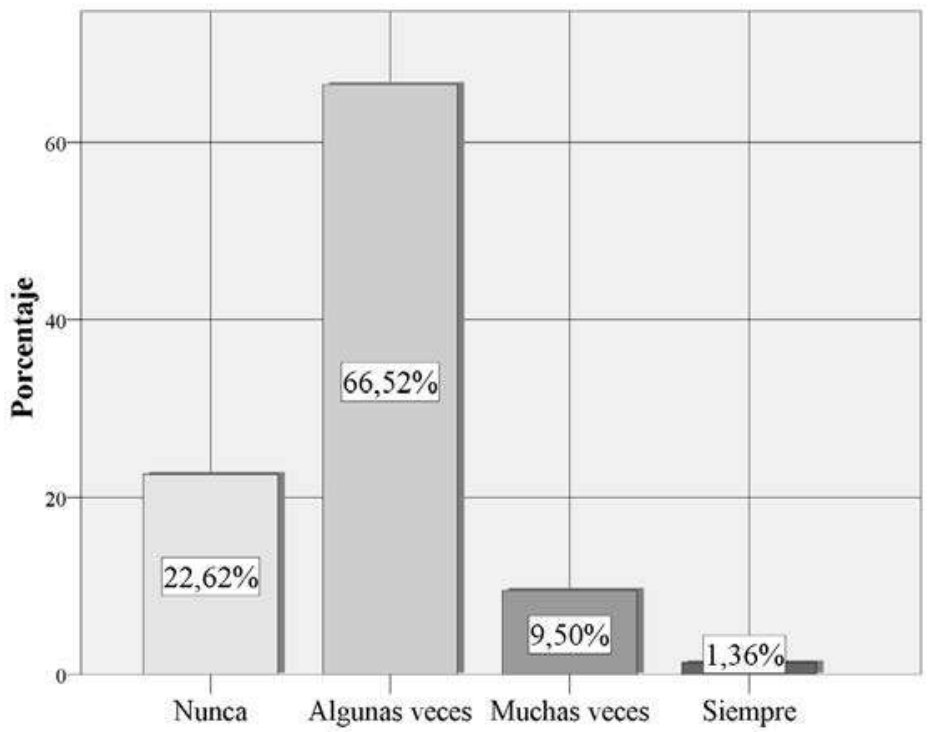

Fuente: elaboración propia a partir de los resultados de la investigación.

El programa, al contemplar la formación para la innovación, debe elaborar su propio plan, y actualizarlo en la medida en que haya cambios en el objeto de estudio, y en el contexto de las organizaciones y las comunidades. El plan debe promover la articulación de la innovación, con la investigación y, por supuesto, con las demás asignaturas del programa, procurando tener presente la interdisciplinariedad como criterio curricular de formación. Adicionalmente debe focalizar la formación en aquellas actividades que fortalecen la productividad, el uso de los recursos, y la generación de valor, y que son propias de la contabilidad administrativa.

R3. ES DP1. "Me parece supremamente pertinente (...) si logramos unir la investigación con la innovación”. R21. ES DP1. “(...) es una herramienta más que tenemos para poder desarrollar la innovación y para poder justificar todo el proceso investigativo". R7. GD PN1. "La contabilidad administrativa genera 
espacios para la innovación, ya que está orientada a la eficiencia, y esta eficiencia es muy valorada por las empresas". R8. GD PN1. "La eficiencia hay que entenderla como mayor productividad, optimización en el uso de los recursos, en la generación de valor, y la innovación permite generar valor”. R1. ES PE3. “(...) la formación en innovación es una asignatura de transformación (...) ¿Qué significa transformación? Que yo no puedo tener un modelo de un plan de formación de innovación, independientemente cual sea el perfil profesional de la persona que sea netamente informativo (...).

\section{IV.4 Participación en la elaboración del plan de formación para la innovación}

En cuanto a la participación en la elaboración del plan de formación para la innovación, el $81,00 \%$ manifiesta que nunca ha participado; el $18,55 \%$, que algunas veces, y el $0,45 \%$, que muchas veces (figura 4 ).

Figura 4. Participación en la elaboración del plan de formación para la innovación.

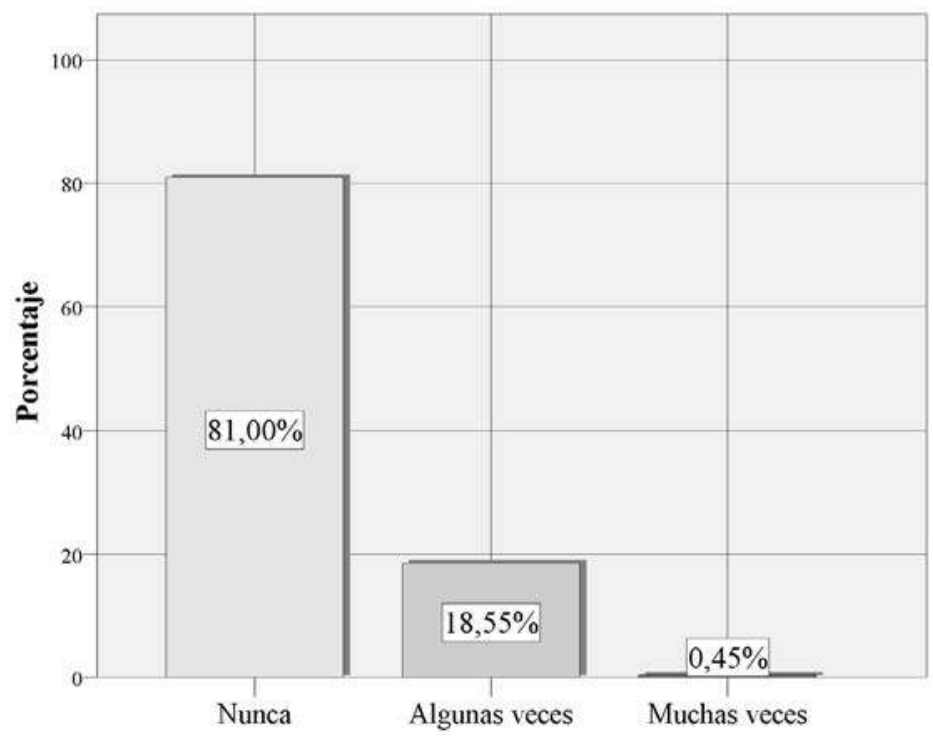

Fuente: elaboración propia a partir de los resultados de la investigación.

Para la elaboración de un plan de formación para la innovación se recomienda contar con la participación de actores involucrados en el proceso. Esto es, por un lado, el programa académico, la empresa y el Estado, y, por otro lado, los directivos, estudiantes y profesores. La innovación en esencia es un ejercicio colectivo, por tanto, se deben consultar los intereses de quienes participan en estos procesos de formación. 
R4. ES DP1. "No, desafortunadamente no, no he tenido ninguna experiencia con este tema (...) creo que viendo todo lo que es la innovación y la importancia que tiene hoy en día la investigación, se abre un gran espacio para poder trabajar en esto”. R13. ES PE3. “(...) hay que trabajar la innovación de cultura, o sea, la innovación en toda la organización y una universidad tiene gente financiera, tiene gente en planeación, tiene gente en los programas (...)” R3. GD PE1. "Para hablar de innovación en las universidades y en los programas académicos, es muy importante conocer las políticas de innovación de las instituciones, ya que no podemos pretender enseñar lo que la institución o el programa no tienen contemplado".

IV.5 Generación de espacios para socializar productos resultantes de la formación para la innovación

El 52,04\% considera que estos espacios nunca se generan; el $43,44 \%$, que se generan algunas veces; el $4,07 \%$, que muchas veces, y el $0,45 \%$, que siempre (figura 5).

Figura 5. Generación de espacios para socializar los productos resultantes de la formación para la innovación.

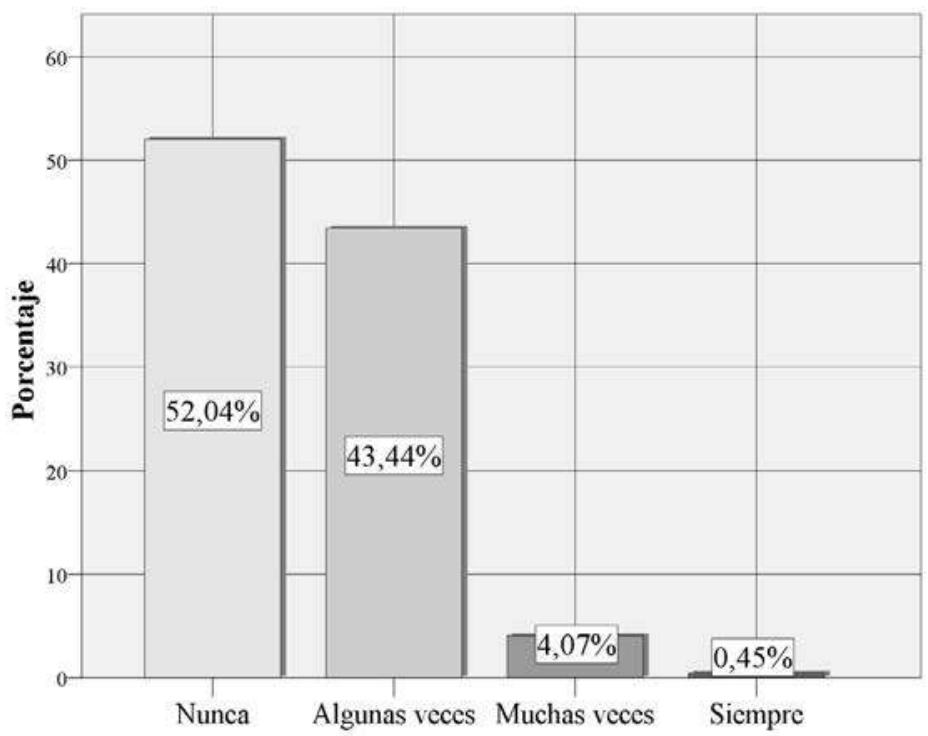

Fuente: elaboración propia a partir de los resultados de la investigación.

Los espacios que se generan para socializar los productos resultantes de la formación para la innovación se dan ocasionalmente, y ello obedece a que, si no hay un proceso de formación para la innovación sólido y posicionado, los productos y espacios para su socialización son limitados o casi inexistentes. 
Algunos de estos espacios se dan solo ocasionalmente en el aula de clase, lo cual lleva a que este ejercicio se convierta en una actividad netamente académica.

R5. ES DP1. “(...) en los comités curriculares hemos dialogado sobre la importancia de innovar los currículos (...) obviamente tenemos que fortalecer la innovación, tema que nos va a permitir mejorar la calidad académica”.

\section{IV.6 Información sobre cómo se lleva a cabo la formación para la} innovación

El 52,04\% considera que nunca se recibe esta información; el $43,44 \%$, que algunas veces, y el 4,52\%, que muchas veces (figura 6).

Figura 6. Recibe información sobre cómo se lleva a cabo la formación para la innovación.

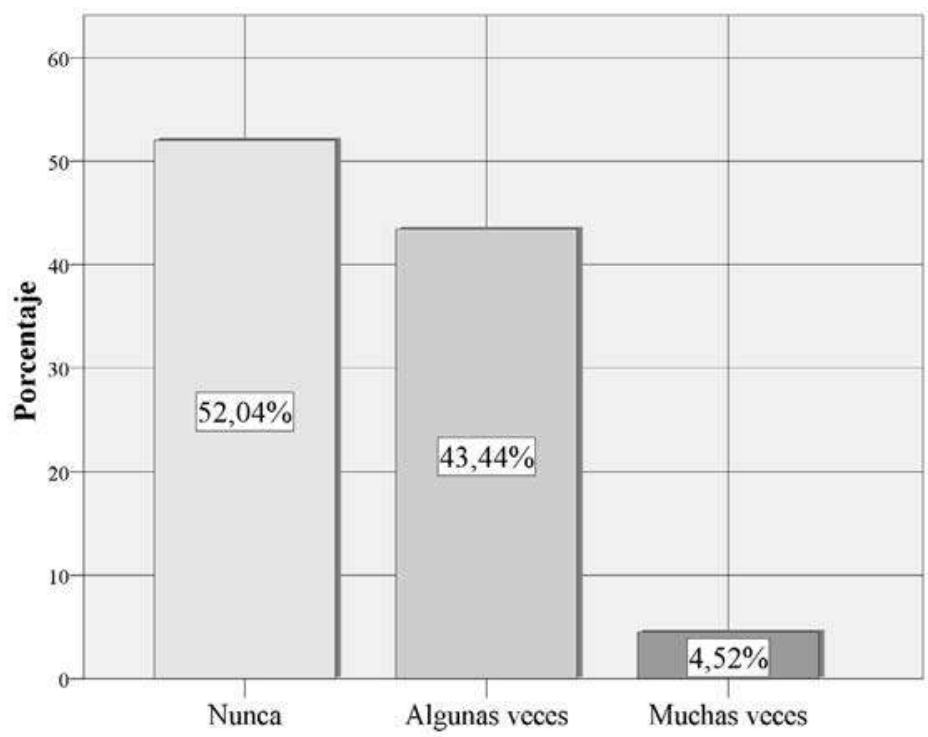

Fuente: elaboración propia a partir de los resultados de la investigación.

La información sobre cómo se lleva a cabo la formación para la innovación aún no es clara para los estudiantes, en consecuencia, se requiere documentar mediante un plan de curso, la forma como se lleva a cabo este proceso y, por supuesto, optimizar los canales de comunicación entre el programa, los estudiantes y los docentes, para que esta información sea de conocimiento general para la comunidad académica.

R10. ES DP1. “(...) el tema de innovación de aquí en adelante es un reto, pues para mí como director y para el programa, para que los estudiantes, los profesores y toda la comunidad académica estemos totalmente vinculados". 
IV.7 Grado de importancia de la formación para la innovación en su condición de estudiante

El 41,63\% considera que la formación para la innovación es bastante importante; el $33,48 \%$, que es muy importante; el $18,55 \%$, que es poco importante, y el 6,33\%, que es nada importante (figura 7).

Figura 7. Grado de importancia de la formación para la innovación en su condición de estudiante.

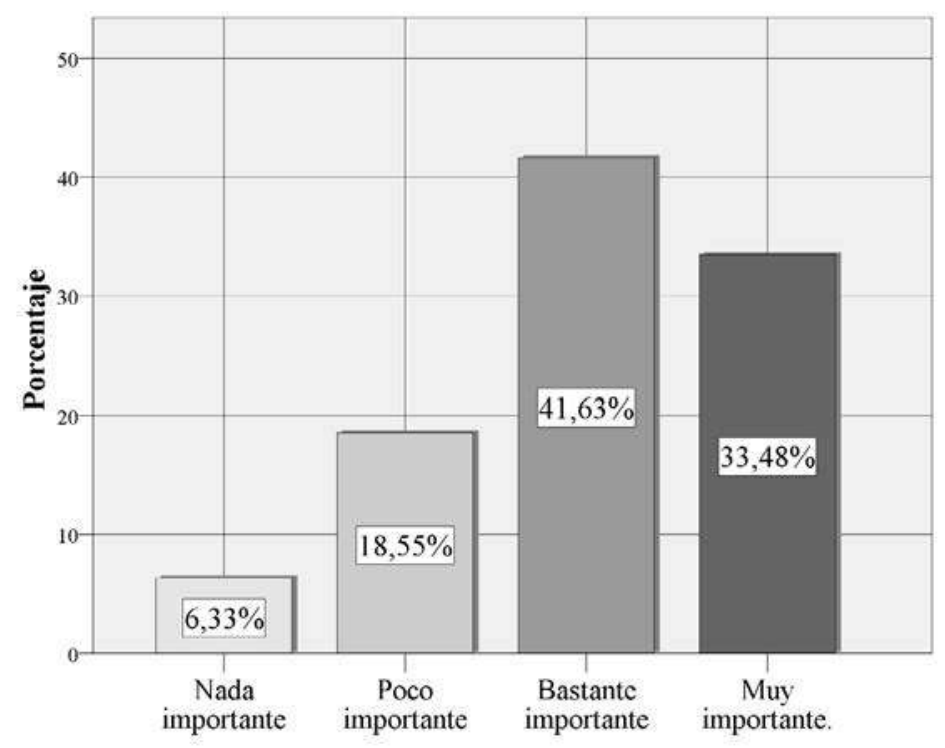

Fuente: elaboración propia a partir de los resultados de la investigación.

Para los estudiantes, la importancia de la formación para la innovación está dada por el hecho de que, en la medida en que aprendan a innovar, este aprendizaje se puede aplicar en cualquier contexto de su vida personal y profesional. Ello se manifiesta en el grado de importancia que asignan a la formación para la innovación en el programa que cursan, lo cual indica que hay una gran expectativa al respecto.

R1. GD GP1. "Para mí el tema ha sido complejo, entender la innovación, sé que hay bastantes teorías y autores, pero la dificultad es la aplicación de estas teorías, es decir cómo innovar en la práctica". R2. GD GP1. "Inicialmente cuando se hablaba de innovación, inmediatamente nos trasladábamos al mundo de la tecnología y en elementos de última tecnología, pero no pensábamos en la innovación como elemento que se enseña y se aprende. R3. GD GP1. "El profesional tiene sus propios momentos y espacios para innovar, y ello está 
relacionado con la toma de decisiones". R4. GD GP1. "Yo innovaría en temas de sistemas de información financiero para toma de decisiones en pymes".

IV.8 La formación para la innovación potencia el rendimiento académico

El 36,65\% manifiesta que la formación para la innovación siempre potencia el rendimiento de los estudiantes; el $35,29 \%$, que muchas veces; el $23,08 \%$, que algunas veces, y el $4,98 \%$, que nunca (figura 8 ).

Figura 8. La formación para la innovación potencia el rendimiento académico.

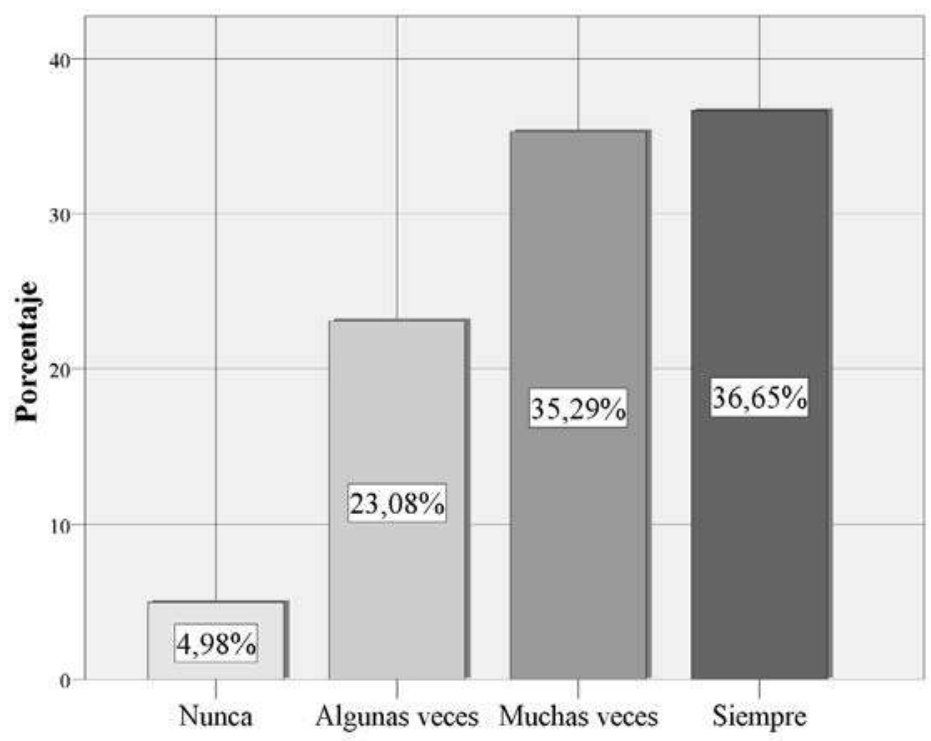

Fuente: elaboración propia a partir de los resultados de la investigación.

La formación para la innovación potencia el rendimiento académico de los estudiantes, puesto que les permite exteriorizar y plasmar su creatividad para generar innovaciones que luego pueden llevar a cabo mediante acciones emprendedoras. Estas acciones innovadoras pueden ser orientadas a la vida académica, mediante formas novedosas de consultar, procesar y comprender la información y, lo más importante, nuevas formas de dar uso óptimo a la información.

R3. GD IN2. "Con las normas internacionales de información financiera, los profesionales hemos tenido que innovar, que desaprender para aprender, el mundo necesita que seamos innovadores". R5. GD IN2. "Los profesionales deben vincularse activamente con los temas de ciencia, tecnología e innovación, ya que ello les permitirá ser actores de los procesos empresariales". 


\section{Conclusiones}

El programa académico tiene como uno de sus propósitos formar profesionales innovadores y transformadores de la realidad social de su contexto; sin embargo, no especifica la forma como se está llevando a cabo esta formación en innovación, lo que explica el bajo grado de conocimiento respecto al modelo de innovación del programa. Tradicionalmente se ha considerado que la innovación es propia de la industria, y no ha tenido un desarrollo pleno en la universidad.

Además, dentro del programa se habla poco de innovación y mucho de investigación. A pesar de ello, hay un gran interés por el tema, lo que implica que se debe socializar con mayor frecuencia y profundidad en materia de innovación en el programa. Esto implica hacer explícitos lo propósitos de formación mediante la actualización de los planes de estudio y planes de curso, así como los métodos, técnicas e instrumentos para aprender a pensar, para aprender a aprender y a hacer.

El programa de Contaduría Pública debe elaborar su propio plan y actualizarlo en la medida en que haya cambios en el objeto de estudio y en el contexto de las organizaciones y las comunidades. El plan debe procurar la articulación de la innovación, con la investigación y, por supuesto, con las demás asignaturas del programa. Para la elaboración del plan se recomienda contar con la participación de actores involucrados en el proceso. Esto es, por un lado, el programa académico, la empresa y el Estado y, por otro lado, los directivos, estudiantes y profesores. La innovación, en esencia, es un ejercicio colectivo, por tanto, se deben consultar los intereses de quienes participan en estos procesos. Los espacios para la innovación se dan ocasionalmente en el aula de clase, lo cual lleva a que este ejercicio se convierta en una actividad netamente académica. En la formación para la innovación, la elaboración de prototipos - ya sea de proceso, producto, servicio o cultura - es fundamental $\mathrm{y}$, en consecuencia, se requiere un viraje hacia el trabajo práctico y la socialización pública de resultados.

Aún no es clara para los estudiantes la información sobre cómo se da la formación para la innovación, por tanto, se requiere documentar mediante un plan de curso la forma como se lleva a cabo este proceso y, por supuesto, optimizar los canales de comunicación entre el programa, los estudiantes y los profesores para que esta información sea de conocimiento general de la comunidad académica. Adicionalmente el profesorado debe ser formado en innovación, especialmente en lo relacionado con espíritu innovador, flexibilidad, trabajo en equipo, conocimientos tecnológicos, confianza en su profesión, y sentido de responsabilidad y compromiso. 
Para los estudiantes, la importancia de la formación para la innovación está dada por el hecho de que, en la medida que aprendan a innovar, este aprendizaje se puede aplicar en cualquier contexto de su vida académica. Ello se manifiesta en la importancia que asignan a la formación para la innovación, que indica que hay una gran expectativa al respecto. La formación para la innovación potencia el rendimiento académico, pues les permite a los estudiantes exteriorizar y plasmar su creatividad para generar innovaciones que luego pueden llevar a cabo mediante acciones emprendedoras. Estas acciones innovadoras pueden ser orientadas a la vida académica, mediante formas novedosas de consultar, procesar, comprender y dar uso óptimo a la información.

Sin embargo, la gestión de la innovación presenta una brecha respecto a las ciencias empresariales. En consecuencia, las facultades de ciencias empresariales, con algunas excepciones, no se han interesado por incluir en sus planes de estudio espacios académicos y proyectos de investigación relacionados con la formación para la innovación y la innovación en sí. Las instituciones educativas son las llamadas a consolidar la cultura de la innovación, y ello se logra mediante procesos de formación adecuados, pertinentes y coherentes en los diversos niveles de la educación. Para el caso de las Instituciones de Educación Superior, además de formar para la innovación en el ejercicio de la docencia, lo pueden hacer mediante la proyección social, esto es vinculando las comunidades con proyectos de innovación en sus respectivos contextos.

La formación para la innovación en el contexto de la universidad y con relación a la empresa, evidencia que no hay articulación entre la academia y el sector productivo, ya que, aunque en la educación universitaria se enseña emprendimiento, esta formación no da los resultados esperados, igualmente sucede con la formación en creatividad e innovación. Los estudiantes universitarios tienen grandes expectativas respecto a la formación para la innovación, pues consideran que formarse en este campo les permite potencia su cualificación académica, pues les permitirá contar con actitudes, habilidades y destrezas, y se genera la posibilidad de aplicarlas en el contexto académico y empresarial.

\section{Referencias bibliográficas}

Álvarez, A., y Rubio, A. (2010). Formación de formadores después de Bolonia. Madrid: Díaz de Santos S.A.

Anguera, M.T. (1995). Métodos de investigación en Psicología. Madrid: Síntesis.

Arraut, L. (2014). Apuntes IE. Una nueva forma de entender el emprendimiento y la innovación. Cartagena de Indias: Innovarraut. Proceso Empresarial.

Bisquerra, R. (1989). Métodos de investigación educativa. Guía práctica. Sabadell: CEAC. Buendía, L. (1997). La investigación por encuesta. En L. Buendía, P. Colás y F. Hernández Pina. Métodos de Investigación en Psicopedagogía. Madrid: McGraw-Hill. 
Cohen, L., y Manion, L. (1990). Métodos de investigación educativa. Madrid: La Muralla.

Colás Bravo, M.P. (1999). Métodos y técnicas cualitativas de investigación en psicopedagogía. En L. Buendía, P. Colás y F. Hernández Pina. Métodos de Investigación en Psicopedagogía. Madrid: McGraw-Hill.

Diccionario de la Lengua Española. Recuperado en agosto 1 de 2016 de http://dle.rae. es/?id=IFIVvz0.

Diccionario de la Lengua Española. Recuperado en agosto 1 de 2016 de http://dle.rae. es/?id=IF1I1Br.

Del Rincón y Otros. (1995). Técnicas de investigación en ciencias sociales. Madrid: Dykinson.

Fox, P. (1986). La innovación y el empresario innovador. La práctica y los principios. Buenos Aires. Editorial Sudamericana, S.A.

Fox, D. (1981). El proceso de investigación en educación. Pamplona: EUNSA.

Gallo, C. (2011). Los secretos de Steve Jobs. Ideas innovadoras que cambiaron el mundo. Bogotá, D.C. Grupo Editorial Norma.

Hamel, G. (2008). El futuro de la administración. Bogotá, D.C. Grupo Editorial Norma.

Hernández Pina, F. (1997). Conceptualización del proceso de la investigación educativa. En L. Buendía, P. Colás y F. Hernández Pina. Métodos de Investigación en Psicopedagogía. Madrid: McGraw-Hill.

Martínez Arias, R. (1995). El método de encuestas por muestreo: conceptos básicos. En M.T. Anguera et al. Métodos de investigación en psicología. Madrid: Síntesis.

Nieto, J. (2012). Y Tú... ¿̇Innovas o Abdicas? Colaborando con la nueva normalidad. Valencia. Editorial Universidad Politécnica de Valencia.

OCDE - EUROSTAT. (2006). Manual de Oslo. Guía para recogida e interpretación de datos sobre innovación. Tercera Edición. Madrid. Grupo Tragsa.

OEI. Organización de Estados Iberoamericanos para la Educación, La Ciencia y la Cultura. Conferencia Científica de la V Cumbre Iberoamericana de Jefes de Estado y de Gobierno. Formación para la Innovación. Conclusiones y recomendaciones. Buenos Aires, Argentina, del 2 al 4 de octubre de 1995.

ONU. Organización de las Naciones Unidas. PUND. Programa de las Naciones Unidas para el Desarrollo. Cumbre para el Desarrollo Sostenible, Agenda 2030 para el Desarrollo Sostenible. ODS. Objetivos de Desarrollo Sostenible. Septiembre de 2015.

Pineda, P., Duran, M., Ciraso, A., Espona, B. (2015). La formación para la innovación en España: situación, características y perspectivas de eficacia. Revista Iberoamericana de Educación. Vol. 67. Número 2.

Ponti, F. (2010). Los 7 movimientos de la innovación. Bogotá, D.C. Grupo Editorial Norma. Robinson, K. (2016). Creative Schools. Penguin Random House Grupo Editorial S.A. Bogotá, D.C., Colombia.

Tejada Fernández, J. (2001). Función docente y formación para la innovación. Edúcame. Revista de la Academia Mexicana de Educación, (4).

Vásquez Rodríguez, F. (2005). Destilar la información. Bogotá, D.C.

Wagner, Tony. (2013). Crear innovadores. La formación de los jóvenes que cambiarán el mundo. Bogotá, D.C. Norma. 